\title{
BUTTERFLY FAUNA (INSECTA, LEPIDOPTERA, PAPILIONOIDEA) OF NORTHERN MOSLAVINA (CROATIA)
}

\author{
ŽELJKA FišTreK \\ Energy Institute Hrvoje Požar, Savska cesta 163, HR-10000 Zagreb, Croatia \\ (e-mail: zfistrek@eihp.hr)
}

Fištrek, Ž.: Butterfly fauna (Insecta, Lepidoptera, Papilionoidea) of northern Moslavina (Croatia). Nat. Croat., Vol. 27, No. 1, 97-109, 2018, Zagreb.

This research into the butterfly fauna of the northern parts of the Moslavina region (northern Moslavina) was conducted between July 2004 and September 2006 with the aim of providing the first information on the butterfly fauna for the area and contributing to the overall knowledge on butterfly species distribution in Croatia. The analysis of samples collected from 13 locations demonstrates the presence of 69 species, which corresponds to $35 \%$ of all species recorded in Croatia. Since the research area is subject to significant anthropogenic influence, this share can be considered as significant. Strictly protected species Lycaena dispar (Haworth, 1802), Euphydryas aurinia (Rottemburg, 1775) Lopinga achine (Scopoli, 1763), Papilio machaon (Linnaeus, 1758) and Parnassius mnemosyne (Linnaeus, 1758) were recorded during the research. Furthermore, the research provided the first data on butterfly fauna of the northern parts of the Moslavina region, and can serve as a basis for further research in the wider region.

Keywords: butterflies, Moslavina, conservation

Fištrek, Ž.: Fauna danjih leptira (Insecta, Lepidoptera, Papilionoidea) sjeverne Moslavine. Nat. Croat., Vol. 27, No. 1, 97-109, 2018, Zagreb.

Istraživanje faune danjih leptira sjevernih predjela Moslavine (sjeverna Moslavina) provedeno je $u$ razdoblju od srpnja 2004. do rujna 2006. godine s ciljem prikupljanja prvih informacija o fauni leptira na navedenom području, te kako bi pridonijelo općem poznavanju rasprostranjenosti leptira u Hrvatskoj. Analiza uzoraka sakupljenih na 13 lokacija ukazuje na prisutnost 69 vrsta što čini $35 \%$ od svih zabilježenih vrsta u Hrvatskoj. Budući da se radi o području koje je pod značajnim utjecajem ljudskih aktivnosti, ovaj udio može se smatrati značajnim. Ovim istraživanjem na navedenom području zabilježene su strogo zaštićene vrste Lycaena dispar (Haworth, 1802), Euphydryas aurinia (Rottemburg, 1775) Lopinga achine (Scopoli, 1763), Papilio machaon (Linnaeus, 1758) i Parnassius mnemosyne (Linnaeus, 1758). Nadalje, istraživanje pruža prve podatke o fauni leptira sjevernih predjela Moslavine, te može poslužiti kao podloga za daljnja istraživanja u široj regiji.

Ključne riječi: leptiri, Moslavina, zaštita

\section{INTRODUCTION}

Butterflies are indicators of changes in the environment due to their specific life cycle and their dependency on larval food plant. Any increase or decrease in their numbers is often a sign of changes in their natural habitat and an indicator of ecological processes (Oostermeijer \& VAN SwaAy, 1998). Numerous human activities such as urbanization, intensive forestry, irrigation, intensive agricultural production with the intensive use of herbicides and pesticides, and abandonment of the traditional way of mowing, are some of the drivers of changes in the composition of butterfly fauna (VAN SwaAy et al., 2010). 
In Croatia, butterflies have been studied since the 19th century. Over the last two decades, a major number of articles have been published in which significant data were given regarding butterfly fauna in Croatia (Мıнос et al., 2007b; ŠAšić \& Miнoci, 2011; SAšıć et al., 2015; Koren et al., 2017), some very rare or new species for Croatian fauna were recorded (Kučinić et al. 1999; Minoci et al., 2005; Miнoci \& ŠAšıć, 2005a; Minoci \& ŠAšić, 2005b; Perković, 2006; Mihoci et al., 2007a; ŠAŠić \& Minoci, 2007; Koren, 2010; Minoci \& ŠAšić, 2009) or a contribution to knowledge about and distribution of a given species was provided (Мiносі \& ŠAšić, 2006; Miносі et al., 2007c; Tvrtкović et al., 2011; Kučinić et al., 2014). Nevertheless, the butterfly fauna of some parts of Croatia has remained unknown due to the lack of systematic research. The northern part of the Moslavina region (northern Moslavina) is one such area in which no research has been conducted. Moslavina is a micro-region situated in central Croatia (Fig. 1) within the territory of three counties. The northern part covers the area from the northern forested slopes of Moslavačka gora (489 m) up to the River Česma and its floodplains (RogLić, 2006). The average elevation of the whole area is between 120-150 meters above sea level. The Moslavina region is characterised by a continental climate and central European vegetation. Fertile soil and rich forest resources have influenced the development of the agriculture (mostly animal husbandry) and forestry sectors, resulting in a significant impact by human intervention into the environment and nature. The objective of this research was to record the butterfly species in semi-natural habitats of northern Moslavina, to create a baseline for further research in this area, and to contribute to knowledge on the distribution of butterfly fauna in Croatia, especially endangered and strictly protected species. In order to understand the potential for species distribution, the diversity of butterfly fauna identified by this research has been compared to available records on butterfly fauna of the other researched areas relatively close to the research area, more precisely the fauna of the surroundings of Karlovac (ŠPANIĆ, 2012) and the fauna of rural parts of Zagreb (Koren et al., 2013).

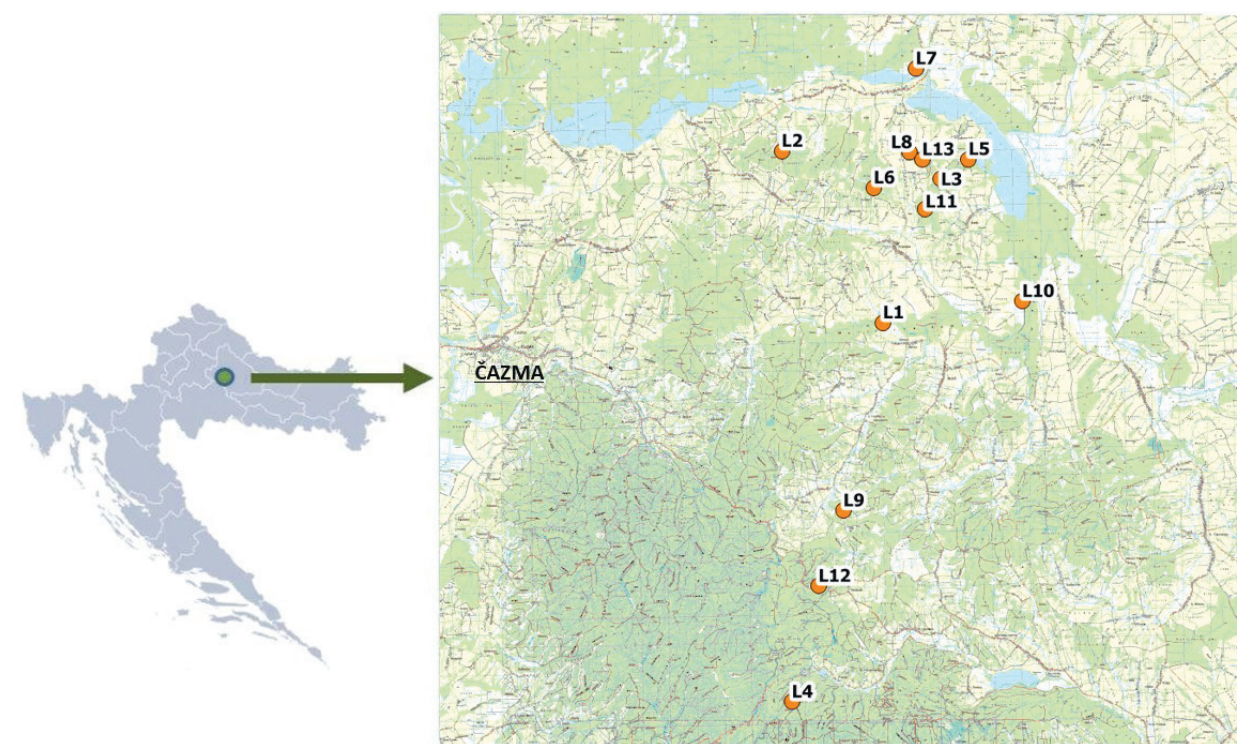

Fig. 1. Position of the research area in Croatia and exact locations of sampling within the northern Moslavina. 


\section{MATERIAL AND METHODS}

The butterflies were collected with an entomological net at 13 locations within the area of northern Moslavina (Fig. 1). The sampling was performed at least once a month from July 2004 to September 2006, during sunny days, between 10 am and 6 pm. Due to the size of the area and the distance between the locations, 2-4 days were needed to visit all the locations. Each location was visited at least 15 times.

When on the spot positive visual identification could be made, butterflies were captured, observed and released, in some cases photographed, while their presence at the location was marked in the research logbook. At the time protected species were not collected. The collected specimens are stored in the author's private butterfly collection.

The altitudes of all collecting sites are between 109 and 356 meters above the sea level (Tab. 1). The sampling locations were chosen based on habitat characteristics, with the aim of achieving greater habitat diversity. The coordinates of the locations, altitudes and types of the habitats are given in Tab. 1. The locations include open patches within dense forest (L12), orchards (L9) and open flooded meadows (L7, L5). All locations have in common significant exposure to anthropogenic influence evident in the presence of agricultural and forestry activities. Two locations (L4 \& L12) are situated within the Moslavačka gora Regional Park while seven locations (L3, L5, L7, L8, L10, L11 \& L13) are within the Natura 2000 site SRA HR1000009 Fishponds along the Česma River. However, the collection of specimens at these locations was conducted prior to the establishment of the Park in 2011 (Regulation on a proclamation of the Moslavačka gora Regional Park, OG 68/11) or the Natura 2000 area in 2013 (Regulation on Ecological Network, OG 124/13). Identification based on wing morphology was made according to ToLMAN \& LEwINGTON (1997). Butterfly nomenclature and systematics follow ŠAšić \& MiносI (2011). Specimens of Leptidea genus, the identification of which is not possible based on the morphology of the wings because of great similarities between species, are identified based on variations in the morphology of their genitalia, which are specific for each species. The analysis of the morphological characteristics of the genitalia was made according to LoRKović (1993), JAKšıć (1998), LELO (2002) and LELO (2003).

Some species of Lycaenidae family were identified by comparison with the entomological collections of the Croatian Natural History Museum (CNHM) in Zagreb.

Tab. 1. List of locations with geographical coordinates (according to Google Earth), altitude and habitat type.

\begin{tabular}{|l|l|l|c|l|}
\hline $\begin{array}{c}\text { Loc. } \\
\text { num. }\end{array}$ & Name & \multicolumn{1}{|c|}{ Coordinates } & $\begin{array}{c}\text { Av. } \\
\text { altitude } \\
(\mathrm{m})\end{array}$ & \multicolumn{1}{c|}{ Habitat type } \\
\hline L1 & Babinac & $45^{\circ} 45^{\prime} 29^{\prime \prime} \mathrm{N}, 16^{\circ} 48^{\prime} 27^{\prime \prime} \mathrm{E}$ & 130 & $\begin{array}{l}\text { Broadleaved deciduous woodland dominated by } \\
\text { beech, oak and hornbeam. Smaller clearings } \\
\text { dominated by shrubs. }\end{array}$ \\
\hline L2 & Blatnica & $45^{\circ} 48^{\prime} 52.2^{\prime \prime} \mathrm{N}, 16^{\circ} 45^{\prime} 27.2^{\prime \prime} \mathrm{E}$ & 119 & $\begin{array}{l}\text { Mesophilous cultivated meadow surrounded by } \\
\text { broadleaved deciduous woodland. }\end{array}$ \\
\hline L3 & Bukvik & $45^{\circ} 48^{\prime} 17^{\prime \prime} \mathrm{N}, 16^{\circ} 50^{\prime} 3.2^{\prime \prime} \mathrm{E}$ & 140 & $\begin{array}{l}\text { Recently felled area in broadleaved deciduous } \\
\text { woodland dominated by beech and oak. Poplar } \\
\text { parches along the stream. }\end{array}$ \\
\hline
\end{tabular}


Tab. 1. Continued

\begin{tabular}{|c|c|c|c|c|}
\hline $\begin{array}{c}\text { Loc. } \\
\text { num. }\end{array}$ & Name & Coordinates & $\begin{array}{l}\text { Av. } \\
\text { altitude } \\
\text { (m) }\end{array}$ & Habitat type \\
\hline L4 & Garić & $45^{\circ} 37^{\prime} 51.7^{\prime \prime} \mathrm{N}, 16^{\circ} 45^{\prime} 41.8^{\prime \prime} \mathrm{E}$ & 356 & $\begin{array}{l}\text { Broadleaved deciduous woodland dominated by } \\
\text { beech. Clearing dominated by shrubs and invasive } \\
\text { vegetation. }\end{array}$ \\
\hline L5 & $\begin{array}{l}\text { Kolarevo } \\
\text { Selo }\end{array}$ & $45^{\circ} 48^{\prime} 43.7^{\prime \prime} \mathrm{N}, 16^{\circ} 50^{\prime} 48^{\prime \prime} \mathrm{E}$ & 115 & $\begin{array}{l}\text { Humid meadows with occasional willow and } \\
\text { poplar trees and smaller shrubs. Reeds at the edges. }\end{array}$ \\
\hline L6 & $\begin{array}{l}\text { Mali } \\
\text { Bjelovar }\end{array}$ & $45^{\circ} 48^{\prime} 4^{\prime \prime} \mathrm{N}, 16^{\circ} 48^{\prime} 9.7^{\prime \prime} \mathrm{E}$ & 131 & $\begin{array}{l}\text { Riparian woodland dominated by oak, poplar and } \\
\text { alder. }\end{array}$ \\
\hline L7 & Narta & $45^{\circ} 50^{\prime} 28.7^{\prime \prime} \mathrm{N}, 16^{\circ} 49^{\prime} 30.4^{\prime \prime} \mathrm{E}$ & 108 & $\begin{array}{l}\text { Humid meadows in succession due to cultivation } \\
\text { abandonment. Individual willow, poplar, alnus } \\
\text { trees and shrubs. Evidence of invasive vegetation. }\end{array}$ \\
\hline L8 & Perinac & $45^{\circ} 49^{\prime} 1.9^{\prime \prime} \mathrm{N}, 16^{\circ} 48^{\prime} 56^{\prime \prime} \mathrm{E}$ & 137 & $\begin{array}{l}\text { Mesophilous cultivated meadow surrounded by } \\
\text { arable land. }\end{array}$ \\
\hline L9 & Samarica & $45^{\circ} 41^{\prime} 50.5^{\prime \prime} \mathrm{N}, 16^{\circ} 47^{\prime} 15.5^{\prime \prime} \mathrm{E}$ & 207 & $\begin{array}{l}\text { Fruit and nut tree orchard. Pasture with mixed } \\
\text { broadleaved deciduous woodland at edges. }\end{array}$ \\
\hline L10 & Srijedska & $45^{\circ} 45^{\prime} 47.6^{\prime \prime} \mathrm{N}, 16^{\circ} 52^{\prime} 20.7^{\prime \prime} \mathrm{E}$ & 114 & $\begin{array}{l}\text { Humid grassland surrounded by riparian } \\
\text { woodland and mosaic of cultivated areas. }\end{array}$ \\
\hline L11 & Sutanjska & $45^{\circ} 47^{\prime} 36.2^{\prime \prime} \mathrm{N}, 16^{\circ} 49^{\prime} 28.1^{\prime \prime} \mathrm{E}$ & 132 & $\begin{array}{l}\text { Humid grassland surrounded by broadleaved } \\
\text { deciduous woodland dominated by oak, horn- } \\
\text { beam, beech. }\end{array}$ \\
\hline L12 & Šimljanik & $45^{\circ} 40^{\prime} 15.2^{\prime \prime} \mathrm{N}, 16^{\circ} 46^{\prime} 1.2^{\prime \prime} \mathrm{E}$ & 239 & $\begin{array}{l}\text { Broad-leaved deciduous woodland dominated by } \\
\text { beech. }\end{array}$ \\
\hline L13 & $\begin{array}{l}\text { Škarcev } \\
\text { Gaj }\end{array}$ & $45^{\circ} 48^{\prime} 41.5^{\prime \prime} \mathrm{N}, 16^{\circ} 49^{\prime} 34.8^{\prime \prime} \mathrm{E}$ & 145 & $\begin{array}{l}\text { Mesophilous cultivated meadow surrounded by } \\
\text { arable land. }\end{array}$ \\
\hline
\end{tabular}

\section{RESULTS}

When the collected samples had been examined, 69 species of butterflies belonging to five butterfly families were identified. The species identified are listed in Tab. 2 according to the systematics by ŠAšić \& Miнoci (2011). Next to the species name, the location and the date of collection or observation are given.

Tab. 2. List of recorded butterfly species in the study area, with location and date of collection or observation

\begin{tabular}{|c|c|}
\hline Species & Location and date of finding \\
\hline \multicolumn{2}{|l|}{ Fam. Papilionidae Latreille, 1802} \\
\hline Parnassius mnemosyne (Linnaeus, 1758) & $\begin{array}{l}\text { Kolarevo Selo, May } 16^{\text {th }}, 2005 \text {; Mali Bjelovar, May } 27^{\text {th }}, 2005 ; \\
\text { Narta, May } 14^{\text {th }}, 2005 ; \text { Srijedska, May } 15^{\text {th }}, 2006\end{array}$ \\
\hline Iphiclides podalirius (Linnaeus, 1758) & $\begin{array}{l}\text { Bukvik, August } 1^{\text {st }}, 2005 \text {; Mali Bjelovar, August 19 } \\
\text { June } 23^{\text {th }}, 2006\end{array}$ \\
\hline Papilio machaon (Linnaeus, 1758) & $\begin{array}{l}\text { Bukvik, August } 1^{\text {st }}, 2005 \text {; Mali Bjelovar, June } 21^{\text {st }}, 2005 \text {; Narta, August } \\
18^{\text {th }}, 2005^{*} \text {; Sutanjska, August } 26^{\text {th }}, 2005 \text {; Škarcev Gaj, August } 1^{\text {st }}, 2005 \text {; } \\
\text { Perinac, August } 16^{\text {th }}, 2006 \text {; Srijedska, July } 20^{\text {th }}, 2006\end{array}$ \\
\hline \multicolumn{2}{|l|}{ Fam. Hesperiidae Latreille, 1809} \\
\hline Erynnis tages (Linnaeus, 1758) & $\begin{array}{l}\text { Bukvik, August } 1^{\text {st }}, 2005 \text {; Sutanjska, August } 2^{\text {nd }}, 2005 \text {; Samarica, } \\
\text { July 20 } 0^{\text {th }}, 2006 \text {; }\end{array}$ \\
\hline Carcharodus alceae (Esper, 1780) & $\begin{array}{l}\text { Narta, August } 18^{\text {th }}, 2005 \text {; Sutanjska, August } 2^{\text {nd }}, 2005 ; \text { Mali } \\
\text { Bjelovar, July } 24^{\text {th }}, 2006 \text {; Samarica, August } 10^{\text {th }}, 2006\end{array}$ \\
\hline
\end{tabular}


Tab. 2. Continued

\begin{tabular}{|c|c|}
\hline Species & Location and date of finding \\
\hline Pyrgus malvae (Linnaeus, 1758) & 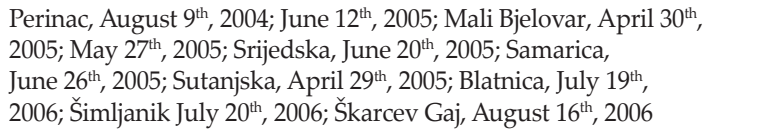 \\
\hline Heteropterus morpheus (Pallas, 1771) & Samarica, August $10^{\text {th }}, 2006$ \\
\hline Thymelicus lineola (Ochsenheimer, 1808) & $\begin{array}{l}\text { Srijedska, June } 27^{\text {th }}, 2005 ; \text { Na rta, August } 18^{\text {th }}, 2005 \text {; Mali Bjelovar, } \\
\text { July } 24^{\text {th }}, 2006\end{array}$ \\
\hline Thymelicus sylvestris (Poda, 1761) & Mali Bjelovar, August 1st, 2005 \\
\hline Ochlodes sylvanus (Esper 1777) & $\begin{array}{l}\text { Samarica, August } 9^{\text {th }}, 2004 \text {; Bukvik, June } 4^{\text {th }}, 2005 ; \text { Kolarevo Selo, } \\
\text { May } 16^{\text {th }}, 2005 \text {; Mali Bjelovar, August } 19^{\text {th }}, 2005^{*} ; \text { Sutanjska, May } 26^{\text {th }} \text {, } \\
2005 \text {; Śimljanik, August } 14^{\text {th }}, 2006\end{array}$ \\
\hline \multicolumn{2}{|l|}{ Fam. Pieridae Swainson, 1820} \\
\hline Leptidea sinapis (Linnaeus, 1758) & 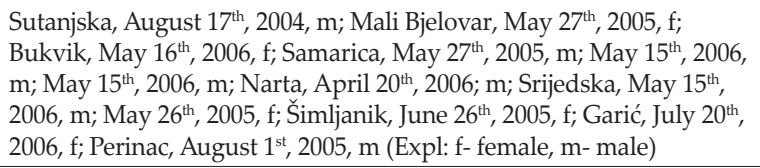 \\
\hline Leptidea juvernica (Williams, 1946) & 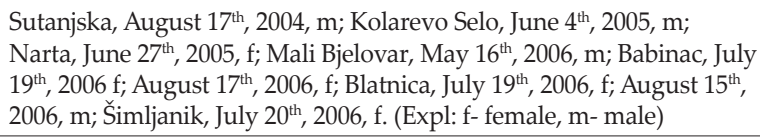 \\
\hline Anthocharis cardamines (Linnaeus, 1758) & 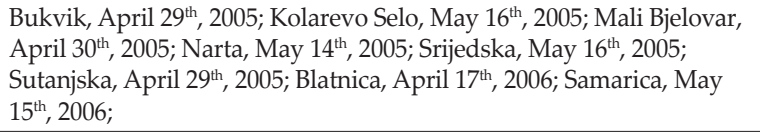 \\
\hline Pieris brassicae (Linnaeus, 1758) & $\begin{array}{l}\text { Perinac, August } 1^{\text {st }}, 2004 ; \text { Bukvik, June } 4^{\text {th }}, 2005 \text {; Babinac, July } 19^{\text {th }} \text {, } \\
\text { 2006; Blatnica, June } 20^{\text {th }}, 2006 \text {; Sutanjska, August } 16^{\text {th }}, 2006\end{array}$ \\
\hline Pieris mannii (Mayer, 1851) & Škarcev Gaj, August $2^{\text {nd }}, 2004 ;$ Blatnica, June $27^{\text {th }}, 2005$ \\
\hline Pieris rapae (Linnaeus, 1758 ) & $\begin{array}{l}\text { Samarica, August } 9^{\text {th }}, 2004 \text {; Mali Bjelovar, August } 16^{\text {th }}, 2004 ; \text { Kolarevo } \\
\text { Selo, June } 4^{\text {th }}, 2005\end{array}$ \\
\hline Pieris napi (Linnaeus, 1758) & 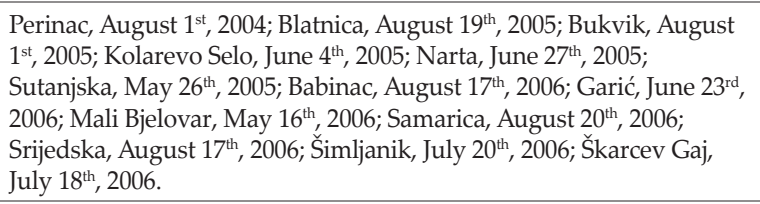 \\
\hline Colias crocea (Geoffroy, 1785) & $\begin{array}{l}\text { Perinac, August } 1^{\text {st }}, 2004 ; \text { Kolarevo Selo, July } 9^{\text {th }}, 2005 ; \text { Narta, } \\
\text { August } 18^{\text {th }}, 2005 \text {; Sutanjska, May } 26^{\text {th }}, 2005 \text {; Samarica, July } 20^{\text {th }} \text {, } \\
\text { 2006; Srijedska, July } 20^{\text {th }}, 2006 \text {; Škarcev Gaj, July } 18^{\text {th }}, 2006\end{array}$ \\
\hline Colias hyale (Linnaeus, 1758) & $\begin{array}{l}\text { Perinac, August } 1^{\text {st }}, 2004 ; \text { Narta, June } 27^{\text {th }}, 2005 \text {; Blatnica, July } 19^{\text {th }} \text {, } \\
\text { 2006; Škarcev Gaj, August } 16^{\text {th }}, 2006\end{array}$ \\
\hline Gonepteryx rhamni (Linnaeus, 1758) & $\begin{array}{l}\text { Samarica, September } 10^{\text {th }}, 2004 \text {; Bukvik, March } 26^{\text {th }}, 2005 \text {; Blatnica, } \\
\text { June } 27^{\text {th }}, 2005 \text {; Mali Bjelovar, April } 30^{\text {th }}, 2005 ; \text { August } 19^{\text {th }}, 2005 \text {; } \\
\text { Srijedska, May } 16^{\text {th }}, 2006 \text {; Sutanjska, May } 26^{\text {th }}, 2005 \text {; Simljanik, May } \\
26^{\text {th }}, 2005 \text {; Babinac, August } 17^{\text {th }}, 2006 \text {; Garić, April } 15^{\text {th }}, 2006 \text {; Narta, } \\
\text { June } 19^{\text {th }}, 2006\end{array}$ \\
\hline \multicolumn{2}{|l|}{ Fam. Riodinidae Grote, 1895} \\
\hline Hamearis lucina (Linnaeus, 1758) & Sutanjska, April 29 ${ }^{\text {th }}, 2005$; Samarica, May $15^{\text {th }}, 2006$; July $20^{\text {th }}, 2006$ \\
\hline \multicolumn{2}{|l|}{ Fam. Lycaenidae Leach, 1815} \\
\hline Lycaena phlaeas (Linnaeus, 1761) & $\begin{array}{l}\text { Samarica, May } 7^{\text {th }}, 2005 \text {; Narta, July } 18^{\text {th }}, 2005 \text {; Škarcev Gaj, } \\
\text { September } 15^{\text {th }}, 2005\end{array}$ \\
\hline
\end{tabular}


Tab. 2. Continued

\begin{tabular}{|c|c|}
\hline Species & Location and date of finding \\
\hline Lycaena dispar (Haworth, 1802) & $\begin{array}{l}\text { Perinac, August } 1^{\text {st }}, 2004 \text {; Samarica, August } 9^{\text {th }}, 2004 \text {; Sutanjska, } \\
\text { August } 17^{\text {th }}, 2004 \text {; Mali Bjelovar, August } 19^{\text {th }}, 2005 \text {; Škarcev Gaj, } \\
\text { August } 1^{\text {st }} \text {, 2005; Kolarevo Selo, June 19 } 9^{\text {th }} \text { 2006; Narta, August } 21^{\text {st }} \text {, } \\
2006\end{array}$ \\
\hline Lycaena tityrus (Poda, 1761) & $\begin{array}{l}\text { Samarica, May } 7^{\text {th }}, 2005 \text {; June } 26^{\text {th }}, 2005 \text {; Babinac, August } 17^{\text {th }}, 2006 \text {; } \\
\text { Mali Bjelovar, May } 16^{\text {th }}, 2006\end{array}$ \\
\hline Lycaena alciphron (Rottemburg, 1775) & $\begin{array}{l}\text { Mali Bjelovar, May 28 } 8^{\text {th }}, 2005 \text {; Samarica, May } 15^{\text {th }}, 2006 \text {; August } 1^{\text {st }} \text {, } \\
\text { 2005; Kolarevo Selo, May } 16^{\text {th }}, 2006\end{array}$ \\
\hline Lycaena hippothoe (Linnaeus, 1761) & $\begin{array}{l}\text { Perinac, August } 1^{\text {st }}, 2004 ; \text { Kolarevo Selo, June } 4^{\text {th }}, 2005 \text {; Samarica, } \\
\text { August } 14^{\text {th }}, 2005 \text {; Narta, August } 18^{\text {th }}, 2005\end{array}$ \\
\hline Thecla betulae (Linnaeus, 1758) & Samarica, August $14^{\text {th }}, 2006$ \\
\hline Callophrys rubi (Linnaeus, 1758) & Samarica, May $7^{\text {th }}, 2005$ \\
\hline Satyrium w-album (Knoch, 1782) & Mali Bjelovar, July $24^{\text {th }}, 2006$ \\
\hline Satyrium pruni (Linnaeus, 1758) & Samarica, May $7^{\text {th }}, 2005$ \\
\hline Cupido argiades (Pallas, 1771) & 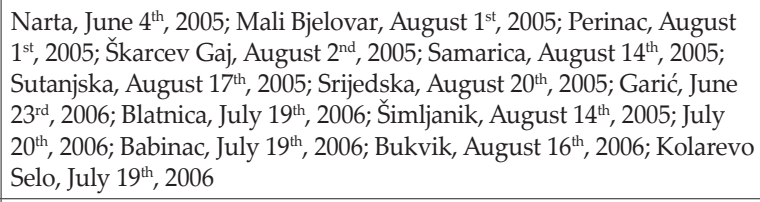 \\
\hline Cupido alcetas (Hoffmannsegg, 1804) & Srijedska, May $15^{\text {th }}, 2006$; Škarcev Gaj, August $16^{\text {th }}, 2006$ \\
\hline Celastrina argiolus (Linnaeus, 1758) & $\begin{array}{l}\text { Garić, August } 11^{\text {th }}, 2005 \text {; Narta, June } 27^{\text {th }}, 2005 \text {; Bukvik, August } 1^{\text {st }} \text {, } \\
\text { 2005; Samarica, May } 27^{\text {th }}, 2005 \text {; Šimljanik, August } 25^{\text {th }}, 2005 \text {; Babinac, } \\
\text { July } 19^{\text {th }}, 2006 \text {; Mali Bjelovar, July } 20^{\text {th }}, 2006 \text {; July } 24^{\text {th }}, 2006 \text {; Srijedska, } \\
\text { July } 20^{\text {th }}, 2006 \text {; Sutanjska June } 4^{\text {th }}, 2006\end{array}$ \\
\hline Polyommatus icarus (Rottemburg, 1775) & 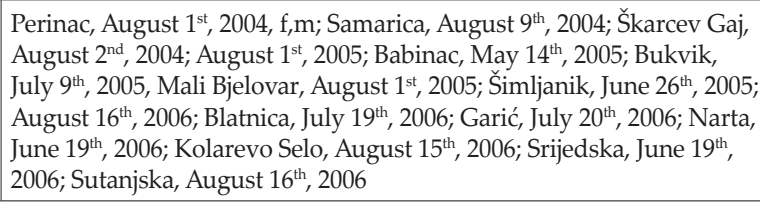 \\
\hline \multicolumn{2}{|l|}{ Fam. Nymphalidae Rafinesque, 1815} \\
\hline Argynnis paphia (Linnaeus, 1758) & $\begin{array}{l}\text { Samarica, August } 9^{\text {th }}, 2004 \text {; Sutanjska, August } 17^{\text {th }}, 2004 ; \text { Babinac, July } \\
10^{\text {th }}, 2005 \text {; Blatnica, August } 19^{\text {th }}, 2005 \text {; Bukvik, August } 27^{\text {th }}, 2005 \text { (f. } \\
\text { valezina); Garić, August } 14^{\text {th }}, 2005 \text {; Narta, June } 27^{\text {th }}, 2005 ; \text { August } 18^{\text {th }} \text {, } \\
\text { 2005; August } 25^{\text {th }}, 2005 ; \text { Mali Bjelovar, August } 1^{\text {st }}, 2005^{*} ; \text { August } 19^{\text {th }} \text {, } \\
\text { 2005; Srijedska, June } 20^{\text {th }}, 2005 \text {; Šimljanik, August } 20^{\text {th }}, 2006\end{array}$ \\
\hline $\begin{array}{l}\text { Argynnis adippe (Denis and Schiffermuller, } \\
1775 \text { ) }\end{array}$ & $\begin{array}{l}\text { Samarica, June } 26^{\text {th }}, 2005 ., \text { Mali Bjelovar, July } 19^{\text {th }}, 2005 \text {; Blatnica, } \\
\text { August } 19^{\text {th }}, 2005\end{array}$ \\
\hline Issoria lathonia (Linnaeus, 1758) & $\begin{array}{l}\text { Perinac, August } 1^{\text {st }}, 2004 \text {; Narta, June } 27^{\text {th }}, 2005 \text {; Srijedska, June } 27^{\text {th }} \text {, } \\
\text { 2005; Blatnica, August } 19^{\text {th }}, 2005\end{array}$ \\
\hline Brenthis daphne (Bergsträsser, 1780) & $\begin{array}{l}\text { Bukvik, June } 4^{\text {th }}, 2005 \text {; Mali Bjelovar, August } 1^{\text {st }}, 2005 ; \text { Narta, June } 27^{\text {th }} \text {, } \\
\text { 2005; Samarica, August } 10^{\text {th }}, 2005 \text {; Srijedska, June } 27^{\text {th }}, 2005 \text {; Sutanjska, } \\
\text { August } 2^{\text {td }}, 2005 \text {, Perinac, June } 4^{\text {th }}, 2006 ; \text { Babinac, June } 23^{\text {rd }}, 2006 \text {; } \\
\text { Garić, July } 20^{\text {th }}, 2006 \text {; Šimljanik, June } 23^{\text {rd }}, 2006\end{array}$ \\
\hline Boloria euphrosyne (Linnaeus, 1758) & Bukvik, July 9 $9^{\text {th }}, 2006$ \\
\hline Boloria dia (Linnaeus, 1767) & Samarica, May 7th, 2005; June $26^{\text {th }}, 2005$ \\
\hline Vanessa atalanta (Linnaeus, 1758) & $\begin{array}{l}\text { Bukvik, October } 3^{\text {rd }}, 2004 ; \text { Kolarevo Selo, June } 4^{\text {th }}, 2005 \text {; Mali Bjelovar, } \\
\text { May 25 } 5^{\text {th }}, 2005 ; \text { Narta, August } 16^{\text {th }}, 2005 \text {; Perinac, June } 25^{\text {th }}, 2005 \text {; } \\
\text { Škarcev Gaj, August } 1^{\text {st }}, 2005 \text {; Samarica, June } 23^{\text {rd }}, 2006\end{array}$ \\
\hline
\end{tabular}


Tab. 2. Continued

\begin{tabular}{|c|c|}
\hline Species & Location and date of finding \\
\hline Vanessa cardui (Linnaeus, 1758) & $\begin{array}{l}\text { Škarcev Gaj, August 2 } 2^{\text {nd }}, 2004 ; \text { July } 4^{\text {th }}, 2004 \text {; Perinac, June } 25^{\text {th }}, 2005 \text {; } \\
\text { Narta, July19 }, 2006\end{array}$ \\
\hline Aglais io (Linnaeus, 1758) & 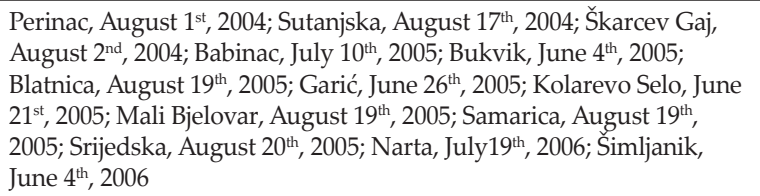 \\
\hline Aglais urticae (Linnaeus, 1758) & $\begin{array}{l}\text { Škarcev Gaj, March } 20^{\text {th }}, 2005 \text {; Perinac, June } 12^{\text {th }}, 2005 ; \\
\text { May } 28^{\text {th }}, 2005\end{array}$ \\
\hline Polygonia c-album (Linnaeus, 1758) & $\begin{array}{l}\text { Samarica, August } 9^{\text {th }}, 2004 ; \text { Perinac, August } 9^{\text {th }}, 2004 ; \text { Babinac, July } \\
10^{\text {th }}, 2005 ; \text { Blatnica, August } 19^{\text {th }}, 2005 ; \text { Bukvik, June } 24^{\text {th }}, 2005 ; \text { Garić, } \\
\text { August } 14^{\text {th }}, 2005 ; \text { Mali Bjelovar, April } 16^{\text {th }}, 2005 ; \text { Narta, June } 27^{\text {th }} \text {, } \\
\text { 2005;Sutanjska, May } 26^{\text {th }}, 2005 \text {, Srijedska, August, 20 } 0^{\text {th }} \text { 2005; Šimljanik, } \\
\text { June, } 26^{\text {th }}, 2005 \text { Garić, July } 20^{\text {th }}, 2006 \text {; }\end{array}$ \\
\hline Araschnia levana (Linnaeus, 1758) & 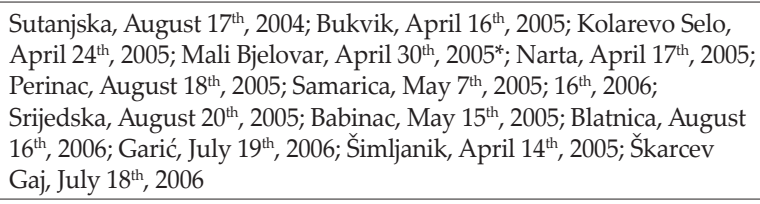 \\
\hline Nymphalis antiopa (Linnaeus, 1758) & $\begin{array}{l}\text { Blatnica, June } 27^{\text {th }}, 2005 \text {; Bukvik, June } 4^{\text {th }}, 2006 \text {; Samarica, May } 27^{\text {th }} \text {, } \\
2006\end{array}$ \\
\hline Nymphalis polychloros (Linnaeus, 1758) & 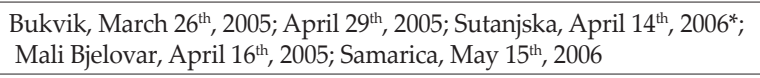 \\
\hline Euphadryas aurinia (Rottemburg, 1775) & Srijedska, July $19^{\text {th }}, 2006$ \\
\hline Melitaea cinxia (Linnaeus, 1758) & Samarica, May $7^{\text {th }}, 2005$; Bukvik, June $4^{\text {th }}, 2005$ \\
\hline $\begin{array}{l}\text { Melitaea phoebe (Denis and Schiffermüller, } \\
\text { 1775) }\end{array}$ & $\begin{array}{l}\text { Perinac, August } 1^{\text {st }}, 2004 \text {; Sutanjska, August 17th }, 2004 \text {; Bukvik, } \\
\text { August } 27^{\text {th }}, 2005\end{array}$ \\
\hline Melitaea didyma (Esper, 1775) & $\begin{array}{l}\text { Sutanjska, May } 26^{\text {th }}, 2005 \text {; Mali Bjelovar, July } 1^{\text {st }}, 2005 \text {; August } 19^{\text {th }} \text {, } \\
\text { 2005; Samarica, August } 14^{\text {th }}, 2005\end{array}$ \\
\hline Melitaea aurelia (Nickerl, 1850) & $\begin{array}{l}\text { Perinac, August } 1^{\text {st }}, 2004 \text {; Mali Bjelovar, August } 1^{\text {st }}, 2005 \text {; Samarica, } \\
\text { June } 23^{\text {rd }}, 2006 \text {; S Simljanik, July } 20^{\text {th }}, 2006\end{array}$ \\
\hline Melitaea athalia (Rottemburg, 1775) & $\begin{array}{l}\text { Sutanjska, August } 17^{\text {th }}, 2004 ; \text { Kolarevo Selo, June } 4^{\text {th }}, 2005 \text {; } \\
\text { Škarcev Gaj, August } 1^{\text {st }}, 2005 ; \text { Bukvik, August } 16^{\text {th }}, 2006\end{array}$ \\
\hline Neptis sappho (Pallas, 1771) & 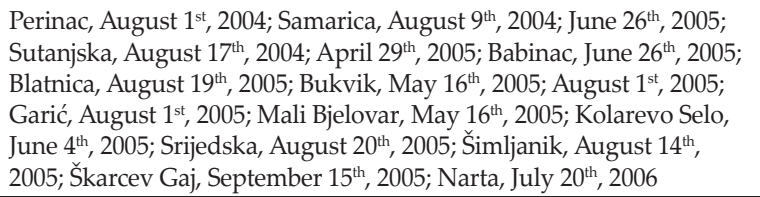 \\
\hline $\begin{array}{l}\text { Apatura ilia (Denis and Schiffermuller, } \\
\text { 1775) }\end{array}$ & $\begin{array}{l}\text { Šimljanik, June } 26^{\text {th }}, 2005 \text {; Blatnica, August } 1^{\text {st }}, 2005 \text {; Mali Bjelovar, } \\
\text { August } 2^{\text {nd }}, 2005\end{array}$ \\
\hline Apatura iris (Linnaeus, 1758) & Šimljanik, June $26^{\text {th }}, 2005$ \\
\hline Pararge aegeria (Linnaeus, 1758) & $\begin{array}{l}\text { Samarica, June } 26^{\text {th }}, 2005 \text {; Bukvik, August } 18^{\text {th }}, 2005 \text {; Sutanjska, } \\
\text { August } 26^{\text {th }}, 2005 \text {; Simljanik, July } 20^{\text {th }}, 2006\end{array}$ \\
\hline Lasiommata megera (Linnaeus, 1758) & $\begin{array}{l}\text { Perinac, September } 12^{\text {th }}, 2004 \text {; Samarica, May } 7^{\text {th }}, 2005 ; \text { Bukvik, } \\
\text { June } 4^{\text {th }}, 2005 ; \text { Mali Bjelovar, August } 1^{\text {st }}, 2005\end{array}$ \\
\hline Lasiommata maera (Linnaeus, 1758 & Samarica, June $26^{\text {th }}, 2005$; July $20^{\text {th }}, 2006$; Sutanjska, August $26^{\text {th }}, 2005$ \\
\hline Lopinga achine (Scopoli, 1763) & $\begin{array}{l}\text { Bukvik, June } 4^{\text {th }}, 2005 \text {; Mali Bjelovar, August 19 } \\
\text { May } 27^{\text {th }}, 2006\end{array}$ \\
\hline Coenonympha arcania (Linnaeus,1758) & Šimljanik, June $26^{\text {th }}, 2005$ \\
\hline
\end{tabular}


Tab. 2. Continued

\begin{tabular}{|c|c|}
\hline Species & Location and date of finding \\
\hline Coenonympha glycerion (Borkhausen, 1758) & $\begin{array}{l}\text { Sutanjska, August } 17^{\text {th }}, 2004 \text {; May } 26^{\text {th }}, 2005 \text {; Perinac, June } 25^{\text {th }} \text {, } \\
\text { 2005; Samarica, June } 26^{\text {th }}, 2005 \text {; Srijedska, August } 20^{\text {th }}, 2005 ; \\
\text { August } 17^{\text {th }}, 2006 \text {; Šimljanik, August } 14^{\text {th }}, 2006\end{array}$ \\
\hline Coenonympha pamphilus (Linnaeus, 1758) & 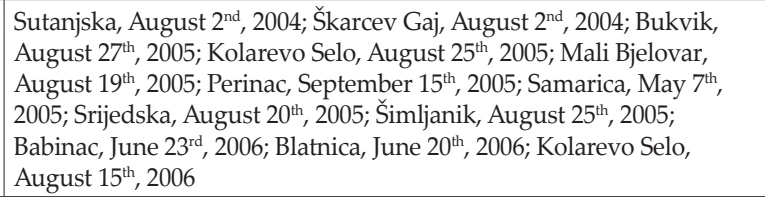 \\
\hline Pyronia tithonus (Linnaeus, 1758) & Samarica, August $9^{\text {th }}, 2004$; Blatnica, August $19^{\text {th }}, 2005$ \\
\hline Aphantopus hyperantus (Linnaeus 1758) & Srijedska, August $17^{\text {th }}, 2006$ \\
\hline Maniola jurtina (Linnaeus, 1758) & 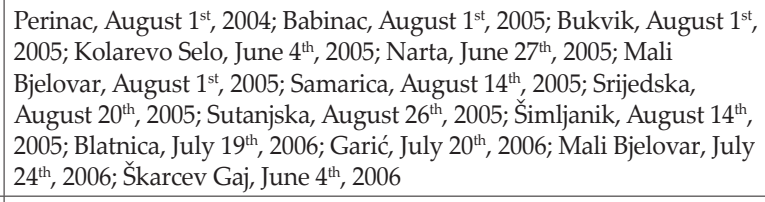 \\
\hline Erebia aethiops (Esper, 1777) & $\begin{array}{l}\text { Mali Bjelovar, August } 1^{\text {st }}, 2005 \text {; Samarica, August 19 }{ }^{\text {th }}, 2005 \text {; Garić, } \\
\text { July } 20^{\text {th }}, 2006\end{array}$ \\
\hline Minois dryas (Scopoli, 1763) & 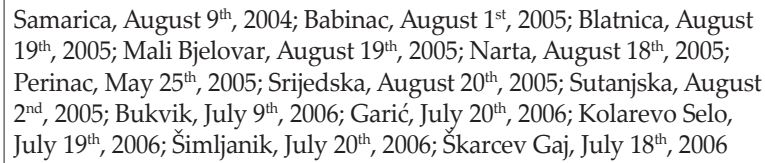 \\
\hline
\end{tabular}

The species that were present in high numbers and were found at all localities are: Pieris napi L., Cupido argiades Pall., Polyommatus icarus Rott., Araschnia levana L., Aglais io L., Argynnis paphia L., Neptis sappho Pall., Coenonympha pamphilus L., Maniola jurtina L., and Minois dryas L. Several species were found only at one of the locations. These are Thecla betulae L. (Samarica, August 14 ${ }^{\text {th }}, 2006$ ), Satyrium w-album Knoch (Mali Bjelovar, July $24^{\text {th }}$, 2006), Satyrium pruni L. (Samarica, May $\left.7^{\text {th }}, 2005\right)$, Callophrys rubi L. (Samarica, May $7^{\text {th }}$, 2005), Apatura iris L. (Šimljanik, June $\left.26^{\text {th }}, 2005\right)$, Boloria euphrosyne L. (Bukvik, July $\left.9^{\text {th }}, 2006\right)$, Boloria dia L. (Samarica, May $7^{\text {th }}, 2005$, June $\left.26^{\text {th }}, 2005\right)$, Euphydryas aurinia Rott. (Srijedska, July $1^{\text {th }}$, 2006), Aphantopus hyperantus L. (Srijedska, August $\left.17^{\text {th }}, 2006\right)$, Coenonympha arcania L. (Šimljanik, May 26 $\left.6^{\text {th }}, 2005\right)$, Thymelicus sylvestris Poda (Mali Bjelovar, August $1^{\text {st }}, 2005$ ), Heteropterus morpheus Pall. (Samarica, August $10^{\text {th }}$, 2006).

The location with the highest number of observed and reported species is L9 - Samarica (47 species), a combination of meadow and an orchard on the sunny slopes of Moslavačka gora which is occasionally grazed by sheep. In fact, locations on forest edges were in general characterised by the highest number of butterfly species. At the localities Samarica, Šimljana, Srijedska several species not found at any other locality were recorded.

The conservation and protection status of the species recorded in northern Moslavina was evaluated according to Red List of Butterflies of Croatia (ŠAšić et al., 2015), and the Ordinance on the proclamation of protected and strictly protected wild taxa (OG 144/13). From 69 recorded species, 9 are listed in the Red List as nearly threatened (NT), while for two species there are insufficient data for estimation of risk of being endangered (DD). From the list, 5 species are recorded in the Ordinance (OG 144/13) and are strictly protected by the Act on Nature Protection (OG 80/13). The conservation actions for butterflies are given in Red Book of Butterflies of Croatia (ŠAšić et al., 2015). The locations with the highest number 
of endangered butterflies are L6, L8 and L9 (Tab. 3). The species Lopinga achine and Euphydryas aurinia were recorded only once.

The analysis of genitalia of cryptic Leptidea genus detected two distinct species, Leptidea sinapis and what was thought to be at the time Leptidea reali. However, the analysis of mitochondrial and nuclear DNA markers of Leptidea genus by DincA et al. (2011) revealed that L. sinapis - L. reali, previously understood to be a cryptic pair, are really part of a triplet of species that also includes L. juvernica. SHтіnкov et al. (2016) indicate that the Leptidea triplet is most likely represented in the Balkans only by L. sinapis and L. juvernica, while L. reali is confined to Iberia, southern France and Italy. Therefore, the specimens identified as L. reali in 2006, are after the revision indicated as L. juvernica.

Tab. 3. Protection status of endangered butterflies recorded in northern Moslavina

\begin{tabular}{|l|c|c|l|}
\hline SPECIES & $\begin{array}{c}\text { National } \\
\text { category }\end{array}$ & $\begin{array}{c}\text { Strictly } \\
\text { protected } \\
\text { (OG 73/16) }\end{array}$ & \multicolumn{1}{|c|}{ Locality } \\
\hline Heteropterus morpheus (Pallas, 1771) & NT & NO & L9 \\
\hline Lycaena dispar (Haworth, 1802) & NT & YES & L6,L8,L9,L11,L13 \\
\hline Lycaena hippothoe (Linnaeus, 1761) & NT & NO & L5,L7,L8,L9 \\
\hline Apartura ilia (Denis \& Schiffermüller, 1775) & NT & NO & L12 \\
\hline Apartura iris (Linnaeus,1758) & NT & NO & L12 \\
\hline Euphydryas aurinia (Rottemburg, 1775) & NT & YES & L10 \\
\hline Lopinga achine (Scopoli, 1763) & NT & YES & L3, L6, L9 \\
\hline Melitaea aurelia (Nickerl, 1850) & DD & NO & L6,L8,L9,L12 \\
\hline Papilio machaon (Linnaeus, 1758) & NT & YES & L7,L11 \\
\hline Parnassius mnemosyne (Linnaeus, 1758) & NT & YES & L5,L6,L10 \\
\hline Pieris brassicae (Linnaeus, 1758) & DD & NO & L1,L2,L3,L8,L11 \\
\hline
\end{tabular}

DD (data deficient), NT (near threatened)

\section{DISCUSSION}

Research into butterflies in the area of northern Moslavina recorded 69 species at 13 localities. This constitutes only 35\% of species of overall butterfly fauna of Croatia, which amounts to 197 species in all (ŠAšić et al., 2015). If this number is compared to a number of species found in geographically, climatically and ecologically similar areas, such as the rural parts of Zagreb (Konen et al., 2013), or the surroundings of Karlovac (ŠPANIć, 2012), it can be assumed that the fauna of northern Moslavina should comprise more species than were found in this research. From 88 species recorded for rural parts of Zagreb, 55 species were also found in northern Moslavina, while some species quite common in the surroundings of Zagreb (found at 4 or more locations) were not found in northern Moslavina. The later include Cupido mimimus, Cupido decoloratus, Plebejus argyrognomon, Polyomatus daphnis from the Lycaenidae family, and Limentis reducta, Melanargia galathea, Hipparichia fagi, and Brintesia circe from the Nymphalidae family. Of the 64 species recorded for the fauna of the surroundings of Karlovac, 53 are also found in northern Moslavina. Species found near Karlovac but not in northern Moslavina, include Carterocephalus palaemon from the Hesperiidae family, Satyrum spini, Satyrum ilicis, Plebeius argus, Plebeius idas from the Lycaenidae family and Brenthis ino, Boloria selene, Melitae diamina, Nepthis rivularis, Brintesia circe and Melanargia galathea 
from the Nymphalidae family. The species Melanargia galathea and Brintesia circe are common species in Karlovac and in the area of rural Zagreb recorded at almost all locations. Carterochepalus palaemon and Satyrium spini were recorded in the environs of both Karlovac and Zagreb, but not in northern Moslavina. The number of species from the Lycaenidae family recorded by this research is surprisingly low (13 sp.). Significantly, more species from the Lycaenidae family (18 sp.) were recorded for the surroundings of Zagreb. Even though these localities are relatively far from northern Moslavina, some common species with wide distributions over continental Croatia could reasonably also be expected in northern Moslavina.

The difference in altitude among the localities is minor and it is unlikely that altitude is one of the factors that defines distribution and structure of butterfly fauna in this area. The differences in composition of butterfly fauna between locations is probably the result of habitat diversity.

Among the collected specimens an intermediate form of the species the European Map Butterfly, Araschnia levana L. (Srijedska, August 20 ${ }^{\text {th }}, 2006$ ) was recorded. This species exhibits a distinct seasonal polyphenism associated with non-overlapping generations that have different developmental pathways (MoreHouse et al. 2012). Intermediate forms, Araschnia levana porima, develop from the long-day larvae or young pupae but at lower temperatures (SAPHIRO, 1976). The different wing pattern formation in the spring- and summer-generation butterflies is proximately triggered by the timing of ecdysteroid release, which is under photoperiodic control and which is mediated by temperature in the pupal stage (Fric et al., 2004).

Agricultural intensification, abandonment of traditional agricultural practices, climate change (including droughts) and change of woodland management are recognised as major threats to butterflies in Europe (VAN SWAAY, 2010), and were recorded at some locations here as well. ŠAšić \& Mıнос (2011) indicate that some species have also suffered major losses in distribution in Croatia and large numbers of butterflies are threatened due to rapid changes in land use. They emphasise the need to identify sites important for such species, which then need to be protected for their survival to be ensured. Eleven recorded species are listed in the Red Book of Butterflies of Croatia. To protect those species, it is important to determine their range of distribution and develop measures of management for these threatened areas. Conservation measures depend on the particular species, but in general include extensive grazing, regular mowing of grasslands, maintenance of forest management etc.

While some localities are well maintained, some are facing changes in the form of either the abandonment of traditional agriculture activities or intensification of farming practice. A potential temporal comparison study of butterfly fauna between the 2006 and today could give us some answers as to how the fauna has changed with the change of habitats.

\section{CONCLUSIONS}

The first survey of butterfly fauna in the north of Moslavina recorded 69 species of butterflies. This number can be considered as significant having in mind that the area of research is subject to significant anthropogenic impact, primarily through agriculture and forestry. Comparison with butterfly fauna of similar and relatively close areas in Croatia, indicates that probably more species should be expected, especially from the Lycaenidae family. To verify this possibility, further research at more localities is needed. 
Eleven species recorded by this research, are listed in the Red Book of Butterflies of Croatia, from which five species are strictly statutorily protected. To strengthen the conservation of threatened species, further research on distribution and population trends is needed that could serve as a foundation for the development of plans, strategies and guidance for conservation.

\section{ACKNOWLEDGEMENTS}

The author wants to express thanks to Dr Mladen Kučinić (Department of Zoology, Faculty of Science, Zagreb) and Jadranka Maras (Energy Institute Hrvoje Požar, Zagreb).

Received May 12, 2016

\section{REFERENCES}

Dinca, V., Lukhtanov, V.A., Talavera, G. \& Vila, R., 2011: Unexpected layers of cryptic diversity in wood white Leptidea butterflies. Nature Communications 2, 324.

Fric, Z., Konvicka, M. \& Zrzavy, J., 2004: Red \& black or black \& white? Phylogeny of the Araschnia butterflies (Lepidoptera: Nymphalidae) and evolution of the seasonal Polyphenizm. Journal of Evolutionary Biology, 17 (2), 265-278.

Hruby, K., 1964: Prodromus Lepidopter Slovenska. Vydavatelstvo Slovensej akademievied, Bratislava, pp. 962.

JAKšıć, P.M., 1998: Male Genitalia of Butterflies of the Balkan Peninsula with a check-list. Slamka, Bratislava, pp. 152.

Koren, T., 2010: First finding of Ripart's Anomalous Blue Polyommatus (Agrodiaetus) ripartii (Freyer, 1830) (Lepidoptera, Lycaenidae) in Croatia. Nat. Croat., 19 (2), 463-467.

Koren, T., Zadravec, M., Štih, A. \& Hlavati, D., 2013: Butterfly fauna (Hesperoidea \& Papilionoidea) of a rural part of Zagreb City, Croatia. Nat. Croat. 22 (2), 253-264.

Koren, T., Lauš, B., Šašić, M., Mihoci, I., Štih, A., Bralić, P. \& Gomboc, S., 2017: Contribution to the knowledge of the butterfly fauna (Lepidoptera: Papilionoidea) of Hrvatsko zagorje, Croatia. Nat. Croat. 26 (2), 167-196. doi:10.20302/NC.2017.26.15

Kučinić, M., TvrtKović, N. \& KLetečKi E., 1999: The False ringlet (Coenonympha oedippus) is a member of the Croatian butterflies fauna after all. Nat. Croat. 8 (4), 399- 405.

Kučinić, M., Randić, M., Mihoci, I., Koren, T., Mrnjavčić, A., Vojvoda, Lauš, B. \& Burić, I., 2014: Contribution to knowledge of the distribution of the Geranium Bronze Cacyreus marshalli (Butler, 1898) (Lepidoptera, Lycaenidae) in Croatia with note on ecology and ethology. Entomol. Croat. 18 (1-2), 49-57.

LELO, S., 2002: Variation in exogenous and endogenus (genitalia) characteristics of butterflies of the species Leptidea sinapis Linnaeus, 1758 (Pieridae, Dismprphiinae) within populations from the area around Sarajevo. Nat. Croat. 11 (3), 293-319.

Lelo, S., 2003: Morfološka varijabilnost vrsta roda Leptidea Billberg, 1820 (Insecta: Lepidoptera, Dismorphiinae) centralnog dela balkanskog poluostrva. Doktorska disertacija. Biološki fakultet. Univerzitet u Beogradu, pp.134.

LoRković, Z., 1993: Leptidea reali Reissinger 1989 (=lorkovici Real 1988) a new European species (Lepid., Pieridae). Nat. Croat. 2 (1), 1-27.

Minoci, I., Tvrtković, N. \& ŠAšıć, M., 2005: Grecian Copper Lycaena ottomanus (Lefebvre, 1830) (Lepidoptera, Lycaenidae)- new species in the Croatian butterfly fauna. Nat. Croat. 14(4), 255-262.

MıносI, I. \& ŠAšıć, M., 2005a: New finding of the butterfly Brenthis ino (Rottemburg, 1775) (Lepidoptera, Nymphalidae) in Croatia. Entomologia Croatica 9(1-2), 77-83.

Mıносı, I. \& ŠAšıć, M., 2005b: New findings of the butterfly Dalmatian Ringlet, Proterebia afra dalmata (Godart, [1824]) (Lepidoptera, Satyrinae) in Croatia. Nat. Croat., 14(2), 121-129.

Minoci, I. \& ŠAšić, M., 2006: New data on the distribution of the Chapman's Blue Polyommatus thersites (Cantener, 1835) (Lepidoptera, Lycaenidae) in Croatia. Entomologia Croatica, 10(1-2), 7-14. 
Minoci, I., Delić, A., GuUrašin, B., Bučar, M. \& Kučinić, M., 2007a: First finding of the critically endangered butterfly Maculinea alcon (Denis \& Schiffermüller, 1775) (Lepidoptera: Lycaenidae) in the Pannonian part of Croatia. Nat. Croat. 16(1), 19-28.

MiносI, I., ŠAšıć, M. \& Vuкović, M., 2007b: Contribution to the butterfly fauna (Hesperioidea \& Papilionoidea) of the Velebit Mountain, Croatia. Nat. Croat. 16(1), 29-62.

Minoci, I., ŠAšić, M. \& TvrTKović, N., 2007c: New data on the distribution of the Croatian endemic butterfly Erebia stirius kleki Lorković, 1955 (Papilionoidea, Nymphalidae, Satyrinae). Nat. Croat. 16 (2), 139-146.

MıносI, I. \& ŠAšić, M., 2009: Occurrence of the satyrine butterfly Lasiommata petropolitana (Fabricius, 1787) confirmed in Croatia. Nat. Croat., 18(1), 175-180.

Morehouse, N.I., Mandon, N., Christides, J.P., Body, M., Bimbard, G. \& Casas, J., 2012: Seasonal selection and resource dynamics in a seasonally polyphenic butterfly J . Evol. Biol. 26 (2013), 175-185.

OfFicial GAZETTE, 2011: Regulation on proclamation of Regional park Moslavačka gora. No. 68/11 (http:// narodne-novine.nn.hr/clanci/sluzbeni/2011_06_68_1484.html)

Official GaZetTe, 2013: Regulation on Ecological Network. No. 124/13 (http://narodne-novine.nn.hr/ clanci/sluzbeni/2013_10_124_2664.html)

Official gazetTe, 2013: Ordinance on the proclamation of protected and strictly protected wild taxa. No. 144/13, 73/16. (http://narodne-novine.nn.hr/clanci/sluzbeni/2013_12_144_3086.html )

Oostermeijer, J.G.B. \& VAN SwaAY, C.A.M., 1998: The relationship between butterfies and environmental indicator values: a tool for conservation in a changing landscape. Biological Conservation 86, Pg. $271 \pm 280$

Perković, D., 2006: Danaus chrysippus (Linnaeus, 1758) (Lepidoptera, Nymphalidae, Danainae), a new species in the fauna of Croatia. Natura Croatica 15(1-2), 61-64.

RogLić, J., 2006: Geografske regije Hrvatske i susjednih zemalja geografske posebnosti i razvojni procesi. Školska knjiga, Zagreb.

SHAPIRO, A.M., 1978: The evolutionary significance of redundancy and variability in phenotypic-induction mechanisms of pierid butterflies (Lepidoptera). Psyche 85 (2-3), 275-283.

Shtinkov, N., Kolev, Z., Vila, R. \& Dinca, V., 2016: The sibling species Leptidea juvernica and L. sinapis (Lepidoptera, Pieridae) in the Balkan Peninsula: ecology, genetic structure, and morphological variation. Zoology 119, 11-20

ŠAšić, M. \& Minoci, I., 2007: New findings of the woodland brown Lopinga achine (Scopoli, 1763) (Lepidoptera: Nymphalidae: Satyrinae) in Croatia. Entomol. Croat. 11 (1-2), $63-67$.

ŠAšıć, M. \& MıносI, I., 2011: Annotated checklist of Croatian butterflies with vernacular names. Natura Croatica, 20(2), 425-436.

ŠAšrć, M., MiHocı, I. \& KučINIĆ, M., 2015: Red book of butterflies of Croatia. Ministry of Environment and Nature Protection, State Institute for Nature Protection \& Croatian Natural History Museum, pp 180. Zagreb.

ŠPanić, R., 2012: Butterfly fauna (Papilionoidea \& Hesperioidea) in the surrounding area of Karlovac, Croatia. Nat. Croat. 21 (2), 455-470.

Tolman, T. \& Lewington, R., 1997: Butterflies of Britain \& Europe. Harper Collins Publishers, pp. 320. London

Tvrtкović, N., Mıнoci, I., \& ŠAšić, M., 2011: Colias caucasica balcanica Rebel, 1901 (Pieridae) in Croatia - the most western distribution point. Nat. Croat. 20(2), 375-385.

Van SwaAy, C., Cuttelod, A., Collins, S., Maes, D., Lopez Munguira, M., Šašić, M., Settele, J., Verovnik, R., Verstrael, T., Warren, M., Wiemers, M. \& Wynhof, I., 2010: European Red List of Butterflies Luxembourg: Publications Office of the European Union (http://ec.europa.eu/environment/nature/ conservation/species/redlist/downloads/European_butterflies.pdf) 


\section{SAŽETAK}

\section{Fauna danjih leptira (Insecta, Lepidoptera, Papilionoidea) sjeverne Moslavine (Hrvatska)}

\section{Ž. Fištrek}

U Republici Hrvatskoj do sada je zabilježeno 197 vrsta danjih leptira (ŠAšić et al., 2015). Terenskim istraživanjem u razdoblju od srpnja 2004. do rujna 2006. na području sjeverne Moslavine zabilježeno je 69 vrsta danjih leptira, odnosno $35 \%$ ukupne faune danjih leptira Hrvatske. Terenska istraživanja na ovom području do sada nisu provođena tako da je ovo prvi cjeloviti popis faune danjih leptira ovog područja. Utvrđene su rijetke i ugrožene vrste koje se nalaze na crvenom popisu leptira Hrvatske: Heteropterus morpheus Pall., Parnassius mnemosyne L., Apatura iris L., Apatura ilia D\&S., Lycaena dispar Haworth., Lycaena hippothoe L., Euphydryas aurinia Rott., Lopinga achine Scop., Heteropterus morpheus Pall., te Mellicta aurelia Nick. Zabilježena je i netipična forma ljetne generacije vrste Araschnia levana L.

Iz priloženih rezultata možemo reći da je fauna danjih leptira sjeverne Moslavine relativno bogata i raznolika vrstama. Međutim, posljednjih godina vidljiv je trend promjene poljoprivredne strukture što potencijalno može utjecati i na faunu leptira. Da bi se mogle poduzeti odgovarajuće mjere potrebno je prvo napraviti inventarizaciju i kartiranje rasprostranjenosti leptira jer se na temelju tih podataka može procijeniti stvarna ugroženost i utvrditi odgovarajuće mjere zaštite. 
